\title{
Prevalência de sintomas depressivos em idosos atendidos em Unidades Básicas de Saúde em um município do estado de Minas Gerais
}

\author{
Prevalence of depressive symptoms in the elderly attended at Basic Health Units in a \\ municipality in the state of Minas Gerais
}

\section{Prevalencia de síntomas depresivos en ancianos atendidos en Unidades Básicas de Salud en un município em el estado de Minas Gerais}

Letícia Silva Santos ${ }^{1}$, Keity Cristina Bueno Perina ${ }^{1 *}$, Virgínia Vitalina de Araújo Lima ${ }^{1}$, Anna Clara Araújo Moreira ${ }^{1}$, Esther Caroline Santos ${ }^{1}$, Sarah Dutra de Resende Vieira ${ }^{1}$, Camila Barreto Tavares ${ }^{1}$.

\section{RESUMO}

Objetivos: Verificar a prevalência de sintomas depressivos, nível de estresse percebido e nível de funcionalidade e incapacidade em idosos assistidos em Unidades Básicas de Saúde. Métodos: Trata-se de um estudo descritivo transversal, com abordagem quantitativa, realizado mediante a coleta dos dados pessoais, sociodemográficos, Mini Exame do Estado Mental (MMEE), aplicação das escalas de depressão geriátrica em versão reduzida (EDG-15), Escala de Estresse Percebido e WHODAS 2.0, em 113 idosos cadastrados em duas Unidades Básicas de Saúde de um município do Estado de Minas Gerais, MG. Resultados: A média de idade foi de 70,36 anos, em uma amostra composta principalmente por mulheres (50,4\%). Dos 113 idosos estudados, 27,5\% apresentaram sintomas depressivos, predominando o sexo feminino (20,4\%) e $83,2 \%$ apresentaram algum grau de incapacidade funcional, sendo que a maioria, $64,6 \%$, apresentava incapacidade funcional leve. Houve associação significativa entre sintomas depressivos e as variáveis: qualidade de sono, ansiedade, nível de estresse percebido, atividade física e desconforto físico. Conclusão: Os resultados indicam a necessidade de estruturação de políticas, ações preventivas e serviços de saúde que contemplem o cuidado integral da pessoa idosa.

Palavras-chave: Envelhecimento, Sintomas depressivos, Funcionalidade.

\begin{abstract}
Objectives: To verify the prevalence of depressive symptoms, perceived stress level and level of functionality and disability in elderly people assisted in Basic Health Units. Methods: This is a cross-sectional descriptive study, with a quantitative approach, carried out through the collection of personal data , sociodemographic, Mini Mental State Examination (MMEE), application of the reduced version geriatric depression scales (EDG15), Perceived Stress Scale and WHODAS 2.0, in 113 elderly registered in two Basic Health Units in a municipality in the state of Minas Gerais, MG. Results: The average age was 70.36 years, in a sample composed mainly of women (50.4\%). Of the 113 elderly people studied, $27.5 \%$ had depressive symptoms, predominantly female $(20.4 \%)$ and $83.2 \%$ had some degree of functional disability, with the majority, $64.6 \%$, having mild functional disability. There was a significant association between depressive symptoms and the variables: sleep quality, anxiety, perceived stress level, physical activity, and physical discomfort. Conclusion: The results indicate the need to structure policies, preventive actions and health services that include comprehensive care for the elderly.
\end{abstract}

Key words: Aging, Depressive symptoms, Functionality.

1Universidade do Estado de Minas Gerais (UEMG), Divinópolis - MG. *E-mail: keity.bueno@gmail.com

SUBMETIDO EM: 3/2020

ACEITO EM: 4/2020

PUBLICADO EM: 7/2020

REAS/EJCH | Vol.Sup.n.49 | e3482 | DOI: https://doi.org/10.25248/reas.e3482.2020

Página 1 de 9 


\section{RESUMEN}

Objetivos: Verificar la prevalencia de los síntomas depresivos, el nivel de estrés percibido y el nivel de funcionalidad y discapacidad en las personas de edad asistidas en las Unidades Básicas de Salud. Métodos: Este es un estudio descriptivo transversal, con un enfoque cuantitativo, realizado a través de la recopilación de datos personales. , sociodemográfico, Mini examen del estado mental (MMEE), aplicación de la versión reducida de escalas de depresión geriátrica (EDG-15), Escala de estrés percibido y WHODAS 2.0, en 113 ancianos registrados en dos Unidades básicas de salud en un municipio en el estado de Minas Gerais, MG. Resultados: La edad promedio fue de 70.36 años, en una muestra compuesta principalmente por mujeres (50.4\%). De las 113 personas mayores estudiadas, el 27.5\% tenía síntomas depresivos, predominantemente mujeres $(20.4 \%)$ y el $83.2 \%$ tenía algún grado de discapacidad funcional, con la mayoría, $64.6 \%$, con discapacidad funcional leve. Hubo una asociación significativa entre los síntomas depresivos y las variables: calidad del sueño, ansiedad, nivel de estrés percibido, actividad física y malestar físico. Conclusión: Los resultados indican la necesidad de estructurar políticas, acciones preventivas y servicios de salud que incluyan atención integral para las personas mayores.

Palabras clave: Envejecimiento, Síntomas depresivos, Funcionalidad.

\section{INTRODUÇÃO}

O envelhecimento é um acontecimento natural, fisiológico, gradual e irreversível, acompanhado por várias alterações funcionais, com alterações psicológicas e motoras, além do aumento na prevalência de doenças (MACIEL MG, 2010; SOUZA WC, et al., 2015). Atualmente, o Brasil apresenta muitas mudanças relacionadas ao perfil demográfico, acompanhando o cenário mundial, sendo considerado o sexto país do mundo em taxa de envelhecimento populacional (MORAES EN, 2012). No Brasil, esse processo vem ocorrendo em alta velocidade e o Sistema de Saúde ainda não conseguiu adaptar-se de forma a gerar qualidade de vida ou atender a população idosa (FIEDLER MMM e PERES KKG, 2008).

Segundo o Instituto Brasileiro de Geografia e Estatística (IBGE), o número de idosos superou a marca dos 30,2 milhões de idosos em 2017. Em 2012, a população idosa era de 25,4 milhões, o que corresponde a um crescimento de $18 \%$. As mulheres são a maioria, com 16,9 milhões ( $56 \%$ dos idosos), enquanto os homens idosos são13,3 milhões (44\% dos idosos). Acredita-se que em 2050, as crianças de 0 a 14 anos representarão $13,15 \%$, enquanto a população idosa alcançará $22,71 \%$ da população total, o que caracteriza a inversão da pirâmide populacional (IBGE, 2018).

Esta transição demográfica influencia na saúde por meio de mudanças no perfil de morbidade e mortalidade da população, levando à necessidade de reorganização dos modelos assistenciais. Assim, é necessário que $o$ atendimento à saúde do idoso seja voltado para a prevenção e identificação de sinais e sintomas característicos do envelhecimento (ALVES DB e BARBOSA MTS, 2010).

O principal impacto negativo do envelhecimento é o aumento da prevalência de doenças crônicas não transmissíveis (DCNT), que são as principais causas de mortalidade e incapacidade no mundo todo (CAMPOLINA AG, et al., 2013; SILVA MR et al., 2018). A depressão está entre as DCNT que mais causam incapacidade e piora a qualidade de vida, sendo a desordem mais comum nessa faixa etária (COHEN R, et al., 2015). No Brasil, 5,8\% da população apresenta sintomas depressivos sendo este um dos maiores problemas de saúde pública do mundo (WHO, 2017). Campolina AG, et al. (2013) relatam que os sintomas depressivos resultam na perda das funções físicas e mentais dos idosos, interferindo nas atividades cotidianas.

A alta prevalência dos sintomas depressivos em idosos, reforça a necessidade de profissionais da saúde treinados e disponíveis para realização desse diagnóstico de forma precoce, para que sejam realizadas intervenções adequadas, visto que o processo de envelhecimento assume características peculiares em cada indivíduo (MORAES EN, 2012). No âmbito da Fisioterapia, os sintomas depressivos podem influenciar na não evolução do paciente, do ponto de vista funcional, por este motivo, é de suma importância reconhecer e lidar com os sintomas depressivos dos pacientes. Além dos sintomas depressivos, deve-se considerar outras 
consequências para a saúde e a qualidade de vida dos idosos como declínio da capacidade funcional, limitação na realização de atividade física, diminuição da mobilidade, isolamento social, perda da autonomia e independência para a execução das atividades de vida diária (AVD) (BALTES PB e SMITH J, 2006).

De acordo com a Classificação Internacional de Funcionalidade (CIF), o termo funcionalidade abrange as funções e estruturas do corpo; atividade e participação social e fatores ambientais. A funcionalidade diz respeito ao uso das estruturas e funções do corpo para o desempenho de atividades de maneira a satisfazer necessidades e desejos de vida, utilizando recursos pessoais em determinados ambientes e culturas. Referese à capacidade de a pessoa cuidar de si mesma e de determinar tarefas e papéis sociais (FARIAS $\mathrm{N} \mathrm{e}$ BUCHALLA CM, 2005)

A avaliação da capacidade funcional dos idosos permite aos profissionais da saúde uma visão mais precisa quanto à severidade das doenças e o impacto de comorbidades. Além disso, a independência na realização das atividades de vida diária (AVDs) é de grande importância, pois envolve questões emocionais, físicas e sociais e, proporciona melhoria da qualidade de vida dos idosos (VEIGA B, et al., 2016). Nesse sentido, compreender os diferenciais do processo de envelhecimento populacional e avaliar os sintomas depressivos nessa população, pode ajudar na formulação de políticas públicas que visem o cuidado à longo prazo das doenças crônicas, bem como a distribuição e gerenciamento de recursos para a promoção da qualidade de vida e para o planejamento de tratamentos fisioterapêuticos mais eficazes.

Diante do exposto, o objetivo do estudo foi verificar a prevalência de sintomas depressivos em indivíduos com idade igual ou superior a 60 anos e identificar os fatores associados em uma amostra de idosos atendidos nas Unidades Básicas de Saúde de Divinópolis, Minas Gerais, Brasil.

\section{MÉTODOS}

Trata-se de um estudo do tipo observacional transversal com abordagem quantitativa. Foi realizado em duas UBS no município de Divinópolis, MG. Os critérios de inclusão foram: idosos cadastrados nas Unidades de Saúde pesquisadas, ter 60 anos de idade ou mais, concordância em participar do estudo, não apresentar comprometimento grave de linguagem ou compreensão que pudesse interferir na veracidade das respostas e apresentar boa cognição, de acordo com o Mini Exame do Estado Mental (MEEM).

A amostra foi selecionada a partir de amostragem de conveniência, não intencional, de acordo com a demanda atendida, entre agosto e outubro de 2019, considerando a dificuldade de seleção aleatória. A amostra foi posteriormente estratificada quanto as variáveis sociodemográficas, nível de incapacidade, presença ou não de sintomas depressivos e demais variáveis pertinentes ao estudo. Foram sujeitos da pesquisa 113 idosos, sendo 56 homens e 57 mulheres. A coleta de dados teve início após a leitura e assinatura do Termo de Consentimento Livre e Esclarecido (TCLE) e consistiu em entrevista estruturada e aplicação de questionários com os idosos. Ocorreu entre os meses de agosto e outubro de 2019, por meio de entrevistas individuais com os idosos.

Foi utilizado um questionário sociodemográfico, elaborado pela equipe de pesquisa. A presença de sintomas depressivos foi avaliada através da Escala de Depressão Geriátrica - versão reduzida (EDG - 15), contendo 15 perguntas com respostas dicotômicas ("sim" ou "não"), com pontuação máxima de 15 pontos (ALMEIDA OP e ALMEIDA SA, 1999). Para o nível de estresse, foi aplicado a Escala de Estresse Percebido com finalidade de identificar o grau com que os indivíduos percebem as situações estressantes (LUFT CDB, et al., 2007). Contém 14 itens com questões que avalia, o estado da saúde, percepção de situação econômica, da memória, da satisfação com a vida e dos acontecimentos negativos.

As respostas variam de 0 a 4 , sendo $0=$ nunca, 1 = quase nunca, $2=$ as vezes, $3=$ quase sempre e $4=$ sempre, com a pontuação de 0 a 56 pontos, sendo que quanto maior a pontuação maior é o nível de estresse percebido. Para a análise da incapacidade foi aplicada a versão brasileira do WHODAS 2.0 da OMS, traduzida e adaptada para o português (SILVEIRA C, et al., 2013). Teve como objetivo de avaliar a incapacidade associada à condição de saúde nos 30 dias que antecedem sua aplicação. Cada item pode receber pontuações de 0 a 4 que representam "nenhuma" a "extrema dificuldade" para realizar determinada AVD. A soma total varia de 0 a 100 pontos, de nenhuma a máxima capacidade. $O$ estado cognitivo foi avaliado pelo Mini Exame do Estado Mental, com ponto de corte de 13. 
Os dados foram tabulados e analisados no software estatístico (Statistical Package for Social Sciences SPSS 13.0® (Chicago, IL, USA). Os dados numéricos foram apresentados como média e desvio padrão e os dados categóricos foram descritos como número absoluto e porcentagem total. A associação entre sintomas depressivos, capacidade funcional, dados sociodemográficos e saúde foi estimada pelo teste qui-quadrado de Pearson. O nível de significância estatística foi definido em $5 \%$ para todos os testes $(p<0,05)$.

A pesquisa foi aprovada pelo Comitê de Ética em Pesquisa da Universidade do Estado de Minas Gerais (parecer $n^{\circ}$ 3.507.049). Os participantes foram informados e esclarecidos sobre os objetivos e procedimentos da pesquisa e a participação foi voluntária atendendo à Resolução 466/2012 do Conselho Nacional de Saúde. $O$ termo de consentimento livre e esclarecido (TCLE) foi assinado pelos participantes.

\section{RESULTADOS}

Esse estudo foi realizado com 113 idosos atendidos em duas Unidades Básicas de Saúde do município de Divinópolis - MG. A média de idade foi de 70,36 $\pm 4,5$ anos variando de 60 a 89 anos, em uma amostra composta principalmente por mulheres $(50,4 \%)$; sendo $54,9 \%$ dos idosos casados, $26,5 \%$ viúvos; $52,2 \%$ não tinham ensino fundamental completo e $48,7 \%$ dos idosos tinham renda de até 1 salário mínimo (Tabela 1). A prevalência de sintomas depressivos foi de $27,5 \%$, sendo $16,8 \%$ de sintomas depressivos leves/moderados e $10,7 \%$ de sintomas depressivos graves. Destes, $20,4 \%$ eram do sexo feminino $(p=0,04), 13,3 \%$ se encontram entre a idade de 60 a 69 anos; $15,9 \%$ não possuem ensino fundamental completo ou não estudaram; $15 \%$ tem renda de 1 salário mínimo; $14,1 \%$ são casados; e 8,9\% são viúvos.

Tabela 1 - Distribuição das características sociodemográficas e associação com os sintomas depressivos.

\begin{tabular}{|c|c|c|c|c|c|}
\hline \multirow[b]{2}{*}{ Variável } & \multirow[b]{2}{*}{$\begin{array}{l}\text { Distribuição da } \\
\text { população }\end{array}$} & \multicolumn{3}{|c|}{ EDG-15 } & \multirow[b]{2}{*}{$p$} \\
\hline & & $\begin{array}{c}\text { Sem } \\
\text { depressão } \\
\text { n (\%) }\end{array}$ & $\begin{array}{c}\text { Depressão } \\
\text { leve/moderada } \\
\mathrm{n}(\%)\end{array}$ & $\begin{array}{c}\text { Depressão } \\
\text { grave } \\
\text { n (\%) }\end{array}$ & \\
\hline \multicolumn{6}{|l|}{ Sexo } \\
\hline Masculino & $56(49,6)$ & $48(42,4)$ & $5(4,4)$ & $3(2,7)$ & \multirow{2}{*}{$0,04^{x}$} \\
\hline Feminino & $57(50,4)$ & $34(31,0)$ & $14(12,4)$ & $9(8,0)$ & \\
\hline \multicolumn{6}{|l|}{ Idade } \\
\hline $60-69$ & $54(47,8)$ & $39(34,5)$ & $9(8,0)$ & $6(5,3)$ & \multirow{3}{*}{0,54} \\
\hline $70-79$ & $42(37,2)$ & $33(29,2)$ & $4(3,5)$ & $5(4,4)$ & \\
\hline$>80$ & $17(15,0)$ & $10(8,8)$ & $2(1,8)$ & $5(4,4)$ & \\
\hline \multicolumn{6}{|l|}{ Escolaridade } \\
\hline $\begin{array}{l}\text { Fundamental } \\
\text { incompleto }\end{array}$ & $59(52,2)$ & $46(40,7)$ & $8(7,1)$ & $5(4,4)$ & \multirow{5}{*}{0,13} \\
\hline $\begin{array}{l}\text { Fundamental } \\
\text { completo }\end{array}$ & $18(15,9)$ & $13(11,5)$ & $2(1,8)$ & $3(2,7)$ & \\
\hline Médio & $20(17,7)$ & $14(12,4)$ & $3(2,7)$ & $3(2,7)$ & \\
\hline Superior & $9(8,0)$ & $7(6,2)$ & $2(1,8)$ & $0(0,0)$ & \\
\hline Não estudou & $7(6,2)$ & $2(1,8)$ & $0(0,0)$ & $5(4,4)$ & \\
\hline \multicolumn{6}{|l|}{ Renda Mensal } \\
\hline Nenhuma & $16(14,2)$ & $10(8,8)$ & $4(3,5)$ & $2(1,8)$ & \multirow{4}{*}{0,78} \\
\hline Até 1 salário & $55(48,7)$ & $38(33,6)$ & $7(6,2)$ & $10(8,8)$ & \\
\hline 1 a 3 salários & $29(25,7)$ & $23(20,4)$ & $3(2,7)$ & $3(2,7)$ & \\
\hline > 3 salários & $13(11,5)$ & $11(9,7)$ & $1(0,9)$ & $1(0,9)$ & \\
\hline \multicolumn{6}{|l|}{ Estado Civil } \\
\hline Solteiro & $6(5,3)$ & $3(2,7)$ & $1(0,9)$ & $2(1,8)$ & \multirow{4}{*}{0,94} \\
\hline Casado & $62(54,9)$ & $46(40,7)$ & $11(9,7)$ & $5(4,4)$ & \\
\hline Separado & $15(13,3)$ & $13(11,5)$ & $0(0,0)$ & $2(1,8)$ & \\
\hline Viúvo & $30(26,5)$ & $20(17,7)$ & $3(2,7)$ & $7(6,2)$ & \\
\hline
\end{tabular}

Fonte: Santos LS, et al., 2019. 
Dos pacientes que apresentam sintomas depressivos (27,5\%), 16,8\% não praticam atividade física; $15,1 \%$ tem uma qualidade de sono ruim; 23,0\% sentem algum desconforto físico; $22,1 \%$ são ansiosos; $15,9 \%$ apresentam elevado nível de estresse percebido e 26,9\% apresentam algum nível de incapacidade, representado mais à frente com a Tabela 2.

Há evidência de associação entre o grau de depressão e as variáveis qualidade de sono (sendo mais frequente em idosos com qualidade de sono ruim, $p=0,02$ ), níveis de estresse percebido (mais frequente em idosos com níveis elevados de estresse percebido, $p=0,00$ ), nível de incapacidade (mais frequente em idosos com algum grau de incapacidade, $p=0,03$ ), ansiedade (mais frequente em idosos ansiosos, $p=0,03$ ), atividade física (mais frequente em idosos que não praticam atividade física, $p=0,04$ ) e a presença de desconforto físico (sendo mais frequente em idosos com algum tipo de dor, $p=0,01$ ).

Com relação ao nível de estresse percebido, a média foi de $16,93 \pm 9,8$, sendo que $71,7 \%$ dos idosos apresentaram algum nível de estresse percebido; destes, $23 \%$ apresentou nível de estresse percebido muito elevado, e em $15,9 \%$ destes pacientes foi observado presença de sintomas depressivos.

Tabela 2 - Atividade física, qualidade do sono, funcionalidade, estresse percebido, desconforto físico e associação com os sintomas depressivos.

\begin{tabular}{|c|c|c|c|c|c|}
\hline \multirow[b]{2}{*}{ Variável } & \multirow[b]{2}{*}{$\begin{array}{c}\text { Distribuição } \\
\text { da } \\
\text { população } \\
\text { n (\%) }\end{array}$} & \multicolumn{3}{|c|}{ EDG-15 } & \multirow[b]{2}{*}{$p$} \\
\hline & & $\begin{array}{c}\text { Sem } \\
\text { depressão } \\
\text { n (\%) }\end{array}$ & $\begin{array}{c}\text { Depressão } \\
\text { leve/moderada } \\
\text { n (\%) }\end{array}$ & $\begin{array}{l}\text { Depressão } \\
\text { grave } \\
\text { n (\%) }\end{array}$ & \\
\hline \multicolumn{6}{|l|}{$\begin{array}{l}\text { Pratica alguma } \\
\text { atividade física }\end{array}$} \\
\hline Sim & $57(50,4)$ & $45(39,8)$ & $9(8,0)$ & $3(2,7)$ & \multirow{2}{*}{0,04} \\
\hline Não & $56(49,6)$ & $37(32,8)$ & $11(9,7)$ & $8(7,1)$ & \\
\hline \multicolumn{6}{|l|}{ Qualidade do sono } \\
\hline Ruim & $36(31,9)$ & $19(16,8)$ & $8(7,1)$ & $9(8,0)$ & \multirow{4}{*}{$0,02^{*}$} \\
\hline Bom & $33(29,2)$ & $27(23,9)$ & $2(1,8)$ & $4(3,5)$ & \\
\hline Muito bom & $9(8,0)$ & $7(6,2)$ & $0(0,0)$ & $2(1,8)$ & \\
\hline Ótimo & $35(31,0)$ & $29(25,7)$ & $5(4,4)$ & $1(0,9)$ & \\
\hline \multicolumn{6}{|l|}{ WHODAS 2.0} \\
\hline Nenhuma dificuldade & $19(16,8)$ & $18(15,9)$ & $1(0,9)$ & $0(0,0)$ & \multirow{4}{*}{$0,03^{\prime}$} \\
\hline Dificuldade leve & $73(64,6)$ & $55(48,7)$ & $9(8,0)$ & $9(8,0)$ & \\
\hline Dificuldade moderada & $16(14,2)$ & $8(7,1)$ & $4(3,5)$ & $4(3,5)$ & \\
\hline Dificuldade Grave & $5(4,4)$ & $1(0,9)$ & $1(0,9)$ & $3(2,7)$ & \\
\hline \multicolumn{6}{|l|}{ Estresse percebido } \\
\hline Baixo nível & $32(28,3)$ & $31(27,4)$ & $1(0,9)$ & $0(0,0)$ & \multirow{4}{*}{$0,00^{\prime}$} \\
\hline Médio nível & $27(23,9)$ & $21(18,6)$ & $6(5,3)$ & $0(0,0)$ & \\
\hline Alto nível & $28(24,8)$ & $22(19,5)$ & $3(2,7)$ & $3(2,7)$ & \\
\hline Muito alto nível & $26(23,0)$ & $8(7,1)$ & $8(7,1)$ & $10(8,8)$ & \\
\hline \multicolumn{6}{|l|}{$\begin{array}{l}\text { Sente algum } \\
\text { desconforto físico }\end{array}$} \\
\hline Sim & $73(64,6)$ & $47(41,6)$ & $13(11,5)$ & $13(11,5)$ & \multirow{2}{*}{$0,01^{*}$} \\
\hline Não & $40(35,4)$ & $35(31,0)$ & $2(1,8)$ & $3(2,7)$ & \\
\hline \multicolumn{6}{|l|}{ Você é ansioso } \\
\hline Sim & $82(72,6)$ & $57(50,4)$ & $12(10,6)$ & $13(11,5)$ & \multirow{2}{*}{$0,03^{*}$} \\
\hline Não & $31(27,4)$ & $25(22,1)$ & $3(2,7)$ & $3(2,7)$ & \\
\hline
\end{tabular}

Fonte: Santos LS, et al., 2019. 
Utilizando o critério de categorização da variável incapacidade funcional em cinco níveis, os seguintes pontos de corte foram definidos: escore zero (nenhuma incapacidade funcional); escores 1 a 12 (incapacidade leve); escores 13 a 22 (incapacidade moderada); escores 23 a 36 (incapacidade grave) 37 a 48 (incapacidade extrema).

A média total de acordo com o WHODAS 2.0 foi de $7,26 \pm 7,09$, sendo os escores de incapacidade funcional maiores em mulheres, na faixa etária de 70-79 anos, nos idosos que apresentavam menor renda e escolaridade. Houve associação significativa entre sintomas depressivos e nível de incapacidade $(0,03 \%)$.

Apesar da média do escore total do WHODAS 2.0 ter sido pequena na população estudada, 16,8\% não apresentavam incapacidade funcional, $64,6 \%$ apresentavam incapacidade leve, 14,2\% apresentavam incapacidade moderada e 4,4\% apresentavam incapacidade grave. Foi possível observar que, dos 113 participantes estudados, $82,6 \%$ apresentaram algum grau de incapacidade funcional.

Entre os seis domínios de incapacidade avaliados, os mais comprometidos foram participação na sociedade, cognição, mobilidade e atividades de vida diária.

\section{DISCUSSÃO}

$\mathrm{Na}$ população do estudo foi possível observar que, aproximadamente $27,5 \%$ dos idosos investigados tiveram alteração na EDG-15, com presença de sintomas depressivos. Corroborando com os resultados apresentados, Ramos GCF, et al. (2015) e Abrantes GG, et al. (2019) também encontraram uma prevalência de sintomas depressivos em $27,5 \%$ e $25 \%$, respectivamente.

Em contrapartida, Silva MR, et al. (2018), Cohen R, et al. (2015), Silva PA, et al. (2019) e Matias AGC, et al. (2016) demonstram uma prevalência de $30,6 \%, 36,5 \%, 37,2 \%$ e $52,6 \%$ de idosos com sintomas depressivos, respectivamente; e Piani MC, et al. (2016) e Mendes-Chiloff CL, et al. (2019) demonstraram uma prevalência bem mais baixa, de $7,1 \%$ e $14,2 \%$, respectivamente.

Essa grande variabilidade pode ser reflexo das características próprias de cada população estudada, como afirma o trabalho realizado por Veras RP e Coutinho ESF (1991), que observaram porcentagens diferentes de sintomas depressivos em bairros com populações que apresentavam condições socioeconômicas distintas.

Houve uma maior prevalência de sintomas depressivos no sexo feminino $(20,4 \%)$, corroborando com alguns estudos que sugerem que esse maior risco de depressão em mulheres está associado à sobrecarga de suas funções, principalmente relacionadas à família (esposa, mãe, educadora, entre outras), da maior taxa de sobrevida, da privação de estrogênio e do isolamento social (LOPES JM, et al., 2015; MENDES-CHILOFF CL, et al., 2018).

Os resultados do estudo mostraram associações importantes entre a qualidade do sono e sintomas depressivos $(p=0,02)$. Dos pacientes com presença de sintomas depressivos $(27,5 \%), 15,1 \%$ tinham uma qualidade de sono ruim, o que corrobora com os achados de Lopes JM, et al. (2015), que relatam que a depressão é quatro vezes mais frequente naqueles idosos com pior qualidade de sono.

Segundo estes autores, os idosos que tomam medicação para dormir, três ou mais vezes por semana, apresentam um maior risco de depressão. Segundo Guimarães LA, et al. (2019), a má qualidade do sono pode levar a prejuízos na saúde e dificuldade nas atividades de vida diária do idoso. Consequentemente, os distúrbios de sono vêm acompanhados de problemas de memória, atenção, concentração e déficit de atenção.

Os resultados do estudo mostraram associações importantes entre o nível de estresse percebido e os sintomas depressivos $(p=0,00)$. De acordo com Santos $E B$, et al (2015), um idoso com maior nível de estresse percebido tem 3,34 vezes mais chances de desenvolver sintomas depressivos $(p=0,01)$.

Foi possível observar que $72,6 \%$ dos idosos são ansiosos, e destes $22,1 \%$ apresentam sintomas depressivos. Houve evidência de associação entre o grau de depressão e ansiedade (mais frequente em idosos ansiosos, $p=0,03$ ). Segundo Barreto MAM, et al. (2015), a ansiedade pode ser considerado um fator de risco para a depressão, sendo muito frequente a ocorrência dessas duas condições entre os idosos. 
Com relação ao desconforto físico, $64,6 \%$ dos idosos relataram algum desconforto, mais prevalente no sexo feminino, $37,2 \%$, e destes, $23 \%$ apresentam sintomas depressivos. Storchi $\mathrm{S}$, et al. (2016) também observaram em seu estudo, que o desconforto físico está associado com uma maior incidência de sintomas depressivos, corroborando com os resultados da presente pesquisa.

Quanto à atividade física, cerca de 49,6\% não realizam atividade física, e destes, 16,8\% apresentam sintomas depressivos, corroborando com Gonçalves et al. (2014), que relatam que a atividade física é importante para o alívio do desconforto físico e melhora da qualidade de vida.

Foi possível observar uma associação entre sintomas depressivos e atividade física, ou o que podemos chamar de idosos insuficientemente ativos $(p=0,04)$. A relação entre exercício físico, saúde, qualidade de vida e envelhecimento vem sendo cada vez mais discutida e analisada cientificamente por profissionais de diferentes áreas (MACIEL MG, 2010; SOUZA WC, et al., 2015). Segundo um estudo de Meta-análise realizado por Schuch FB, et al. (2018), baseado em dados de mais de 265 mil pessoas de 20 países diferentes, foi possível concluir que, independente da idade ou localização geográfica, a atividade física funciona como prevenção da depressão. Isso reforça que a inatividade física pode contribuir para o surgimento de sintomas depressivos em indivíduos idosos.

A prevalência de incapacidade funcional na presente pesquisa, para pelo menos uma atividade, foi de $83,2 \%$, onde a maioria $(64,6 \%)$, apresentava incapacidade funcional leve. Destes, $16 \%$ apresentavam sintomas depressivos. Segundo Nóbrega IRAP, et al. (2015), a diminuição na capacidade funcional está diretamente relacionada com a presença de depressão. Segundo os autores, idosos com depressão apresentam maiores comprometimentos físicos, sociais e funcionais, interferindo na qualidade de vida com consequente redução ou perda da independência funcional.

A média total de acordo com o WHODAS 2.0 foi de 7,26 $\pm 7,09$, sendo os escores de incapacidade funcional maiores em mulheres, na faixa etária de 70-79 anos e nos idosos que apresentavam menor renda e escolaridade. Apesar da média do escore total do WHODAS 2.0 ter sido pequena na população estudada, $83,2 \%$ dos idosos apresentaram algum grau de incapacidade funcional. Corroborando com os resultados da presente pesquisa, Veiga B, et al. (2016) também relatam um elevado nível de incapacidade funcional, mais prevalente em mulheres.

Segundo os autores, esses resultados demonstram a necessidade de a equipe de saúde aprimorar o cuidado a essa população, priorizando a manutenção da autonomia e funcionalidade. Estudos mostram que mulheres apresentam maior expectativa de vida que os homens, porém esses anos a mais também são vividos com piores condições de saúde e com maior incapacidade funcional (CAMARGOS MCS, et al., 2008).

Entre os seis domínios de incapacidade avaliados, os mais comprometidos foram cognição, mobilidade e atividades de vida diária. Um achado interessante foi que entre os participantes, apenas 10,6\% classificaram sua saúde como ruim ou muito ruim, porém $18,6 \%$ dos idosos do estudo referiram que suas dificuldades avaliadas com o WHODAS 2.0 interferem gravemente ou extremamente em suas vidas. Estudos têm demonstrado que uma pior percepção de saúde está associada à maior mortalidade, independente de outros fatores de risco para a saúde, incapacidade funcional e condições socioeconômicas (FIEDLER MMM, et al., 2008).

Os resultados do estudo mostraram associações importantes entre os sintomas depressivos e incapacidade funcional. Um idoso com sintomas depressivos tem 8,44 vezes mais chances de desenvolver incapacidade funcional $(p=0,03)$. Segundo Mendes-Chiloff MMM, et al. (2019), a idade isolada, não aumenta o risco de depressão, no entanto, os efeitos da idade na sintomatologia depressiva podem ser atribuídos aos problemas de saúde e incapacidade frequentes em idosos. Scott KM, et al. (2009) afirmam que os transtornos mentais têm uma maior associação com incapacidade funcional do que as morbidades físicas.

Assim, e de acordo com os resultados aqui apresentados, parece fundamental que os profissionais de saúde venham a incorporar ações frequentes, para uma identificação o mais precoce possível de sinais e sintomas depressivos, o que permitiria o desenvolvimento e adoção de medidas estratégicas adequadas à prevenção do aparecimento ou agravamento de quadros depressivos na população idosa. 


\section{CONCLUSÃO}

Foi possível concluir que a prevalência de sintomas depressivos em idosos na comunidade foi de $27,5 \%$, sendo mais frequente no sexo feminino $(20,4 \%)$. Houve associações importantes entre a qualidade do sono e sintomas depressivos, mostrando que $15,1 \%$ dos idosos com sintomas depressivos apresentavam uma pior qualidade de sono. Tendo em vista que o envelhecimento da população assume cada vez maior relevância na sociedade e que esse envelhecimento muitas vezes está associado a presença de sintomas depressivos, a estruturação de políticas e serviços de saúde que comtemplem o cuidado integral da pessoa idosa em conjunto com profissionais de saúde treinados para realizar ações de prevenção, tornam-se de extrema importância para melhorar qualidade de vida do idoso, além de incentivar os idosos para a prática regular de atividades físicas.

\section{REFERÊNCIAS}

1. ABRANTES GG, et al. Sintomas depressivos em idosos na atenção básica à saúde. Revista Brasileira de Geriatria e Gerontologia, 2019; 22(4): 1-7; e.190123.

2. ALMEIDA OP e ALMEIDA SA. Confiabilidade da versão brasileira da Escala de Depressão Geriátrica (GDS) versão reduzida. Arquivo de Neuro-psiquiatria, 1999; 57(2B): 421-426.

3. ALVES DB e BARBOSA MTS. Desigualdades na Mortalidade por doenças Crônicas entre idosos e sua Associação com Indicadores Socioeconômicos no Brasil. Revista Brasileira de Ciências do Envelhecimento Humano, 2011; 7(1): 22-33.

4. BARRETO MAM, et al. As consequências da diminuição de dopamina produzida na substância nigra: uma breve reflexão. Interfaces Científicas - Saúde e ambiente, 2015; 4(1): 83-90.

5. CAMPOLINA AG, ADAMI F, SANTOS JLF. Transição de Saúde e as Mudanças na expectativa de Vida Saudável da População Idosa: Possíveis Impactos da Prevenção de Doenças Crônicas. Cadernos de Saúde Pública, 2016; 29(6): 1217-1229.

6. BALTES PB e SMITH J. Novas fronteiras para o futuro do envelhecimento: a velhice bem-sucedida do idoso jovem aos dilemas da quarta idade. A Terceira Idade, 2006; 17(36): 7-31.

7. CAMARGOS MCS, MACHADO CJ, RODRIGUES RN. Life expectancy among elderly Brazilians in 2003 according to different levels of functional disability. Cadernos de Saúde Pública, 2008; 24(4): 845-852.

8. CHILOFF CLM, et al. Sintomas depressivos em idosos do município de São Paulo, Brasil: prevalência e fatores associados (Estudo SABE). Revista Brasileira de Epidemiologia, 2018; 21(2): 1-16.

9. COHEN R, PASKULIN LMG, PRIEB RGG. Prevalência de Sintomas Depressivos entre idosos em um Serviço de Emergência. Revista Brasileira de Geriatria e Gerontologia, 2015; 307-317.

10. FARIAS N e BUCHALLA CM. A classificação internacional de funcionalidade, incapacidade e saúde da organização mundial da saúde: conceitos, usos e perspectivas. Revista Brasileira de Epidemiologia, São Paulo, 2005; 8(2): 187193.

11. FIEDLER MMM e PERES KKG. Capacidade Funcional e Fatores associados em Idosos do Sul do Brasil: Um Estudo de Base Populacional. Cadernos de Saúde Pública, 2008; 24(2): 409-415.

12. GONÇALVES AK, et al. Qualidade de vida e sintomas depressivos em idosos de três faixas etárias praticantes de atividade física. Revista Kairós Gerontologia, São Paulo, 2017; 17(3): 79-94.

13. GUIMARÃES LA, et al. Sintomas depressivos e fatores associados em idosos residentes. Ciências e Saúde Coletiva, 2019; 24(9): 3275-3282.

14. INSTITUTO BRASILEIRO DE GEOGRAFIA E ESTATÍSTICA (IBGE). Disponível em: http: //www.ibge.gov.br/home/ presidencia/noticias/25072002pidoso.shtm. Acesso em: 16 abril 2020.

15. LOPES JM, et al. Associação da depressão com as características sociodemográficas, qualidade do sono e hábitos de vida em idosos do Nordeste brasileiro: estudo seccional de base populacional. Revista Brasileira de Geriatria e Gerontologia, 2015; 18(3): 521-531.

16. LUFT CDB, et al. Versão brasileira da Escala de Estresse Percebido: Tradução e validação para idosos. Revista de Saúde Pública, 2007; 41(4): 606-15.

17. MACIEL MG. Atividade física e funcionalidade do idoso. Motriz: Revista de Educação Física, 2010; 16(4): $1024-32$.

18. MADEIRA TCS, et al. Depressão em idosos hipertensos e diabéticos no contexto da atenção primária em saúde. Revista APS, 2013; 16(4): 393-398.

19. MATIAS AGC, et al. Indicadores de depressão em idosos e os diferentes métodos de rastreamento. Einstein, 2016; 14(1): 6-11.

20. MORAES EN. Atenção à saúde do idoso: Aspectos Conceituais. Oms, 2012; 102.

21. PIANI MC, et al. Prevalência de sintomas depressivos em idosas de um centro de referência de Atenção ao Idoso no município de Passo Fundo, Rio Grande do Sul. Revista Brasileira de Geriatria e Gerontologia, 2016; $19(6)$ : 930 - 938.

22. RAMOS GCF, et al. Prevalência de sintomas depressivos e fatores associados em idosos no norte de Minas Gerais: um estudo de base populacional. Jornal Brasileiro de Psiquiatria, 2015; 64(2): 122-31.

23. SANTOS EB, et al. Estresse Percebido nos idosos sobreviventes do AVC após a alta hospitalar para a casa. Revista da Escola de Enfermagem da USP, 2015; 49(5): 797-803. 
24. SCHUCH FB, et al. Physical Activity and Incident Depression: A Meta-Analysis of Prospective Cohort Studies. The American Journal of Psychiatry, 2018; 175(7): 631-648.

25. SCOTT KM, et al. Mental-physical co-morbidity and its relationship with disability: results from the World Mental Health Surveys. Psychological Medicine, 2009; 39(1): 33-43.

26. SILVA MR, et al. Sintomas depressivos em idosos e sua relação com dor crônica, doenças crônicas, qualidade do sono e nível de atividade física. Brazilian Journal of Pain, 2018; 1(4): 293-298.

27. SILVA PA, et al. Prevalência de sintomas depressivos e seus fatores associados em idosos atendidos por um centro de referência. Revista Brasileira de Geriatria e Gerontologia, 2019; 22(5): e190188.

28. SILVEIRA C, et al. Adaptação transcultural da Escala de Avaliação de Incapacidades da Organização Mundial de Saúde (WHODAS 2.0) para o Português. Revista da Associação Médica Brasileira, 2013; 59(3): 234-240.

29. SOUZA WC, et al. Exercício físico na promoção da saúde na terceira idade. Saúde Meio Ambiente, 2015; 4(1): 5565.

30. STORCHI S, et al. Qualidade de vida e sintomas de ansiedade e depressão em idosas com e sem dor musculoesquelética crônica. Revista dor, 2016; 17(4): 283-288.

31. VERAS RP e COUTINHO ESF. Estudo de prevalência de depressão e síndrome cerebral orgânica na população de idosos, Brasil. Revista de Saúde Pública, v.25, n.3, p.209-17, 1991.

32. VEIGA B, et al. Avaliação de funcionalidade e incapacidade de idosos longevos em acompanhamento ambulatorial utilizando a WHODAS 2.0. Revista Brasileira de Geriatria e Gerontologia, 2016; 19(6): 1015-1021.

33. WORLD HEALTH ORGANIZATION (WHO). Depression and other common mental disorders: global health estimates. Geneva, 2017. 